\title{
Regards croisés sur une église rurale : Saint-Jean l'Évangéliste de Bard-le-Régulier (Côte-d'Or)
}

\section{Gaëlle Pertuisot}

\section{(2) OpenEdition \\ 1 Journals}

Édition électronique

URL : https://journals.openedition.org/cem/15691

DOI : 10.4000/cem. 15691

ISSN : 1954-3093

Éditeur

Centre d'études médiévales Saint-Germain d'Auxerre

Référence électronique

Gaëlle Pertuisot, «Regards croisés sur une église rurale : Saint-Jean l'Évangéliste de Bard-le-Régulier (Côte-d'Or) », Bulletin du centre d'études médiévales d'Auxerre | BUCEMA [En ligne], 22.2 | 2018, mis en ligne le 23 janvier 2019, consulté le 22 septembre 2022. URL : http://journals.openedition.org/cem/ 15691 ; DOl : https://doi.org/10.4000/cem. 15691

Ce document a été généré automatiquement le 22 septembre 2022.

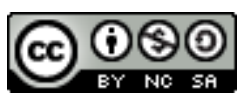

Creative Commons - Attribution - Pas d'Utilisation Commerciale - Partage dans les Mêmes Conditions 4.0 International - CC BY-NC-SA 4.0

https://creativecommons.org/licenses/by-nc-sa/4.0/ 
Regards croisés sur une église rurale : Saint-Jean l'Évangéliste de Bard-le-Régulier (Côte-d'Or)

Gaëlle Pertuisot

Contexte historique et architectural 
1 Le village de Bard-le-Régulier est situé en Côte-d'Or, à l'interface entre Auxois et Morvan, dans une zone rurale. L'habitat, peu dense, y est dispersé le long d'une route départementale (fig. 1 ).

2 Seule l'église se détache du paysage environnant par son aspect massif et par la particularité de son élégant clocher octogonal à deux registres superposés de

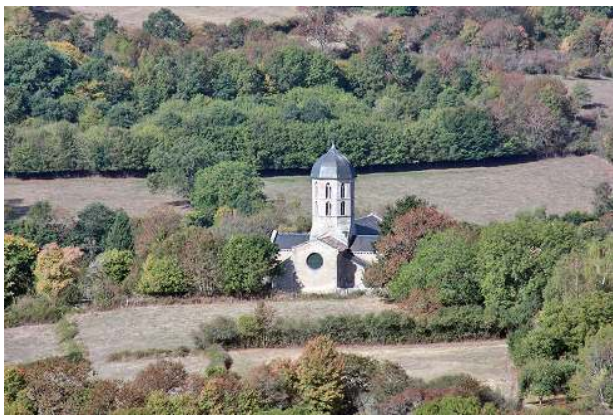
baies géminées (fig. 2).

Fig. 1 - Le village de Bard-le-Régulier et la situation de l'église Saint-Jean l'Évangéliste
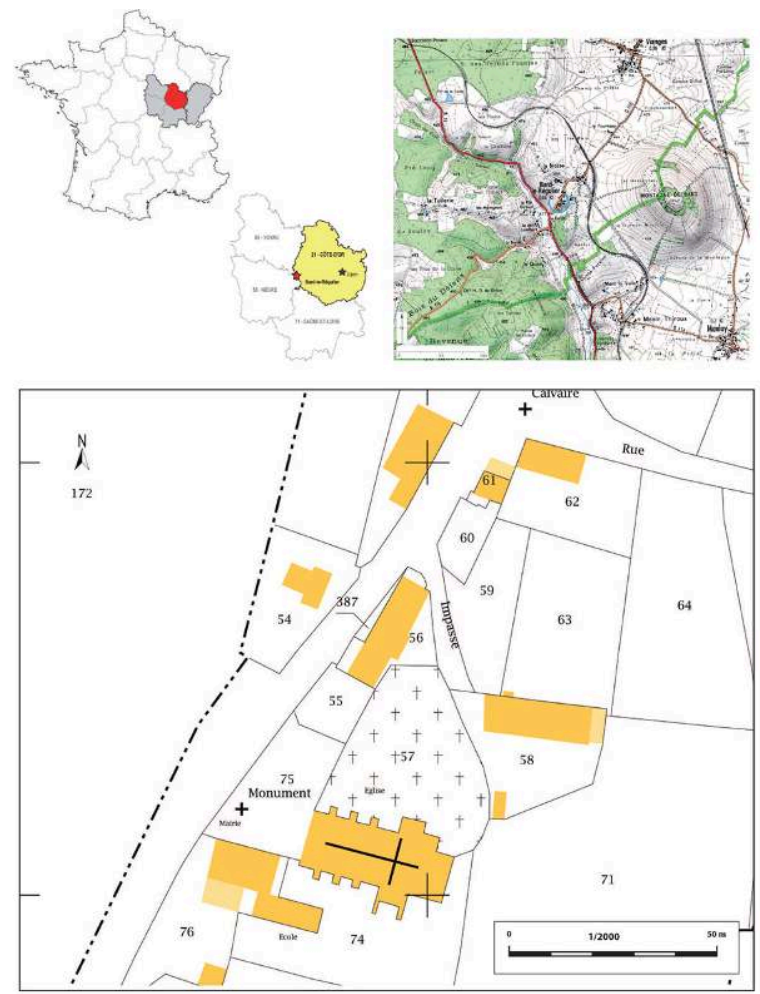

Cl. G. Pertuisot 
Fig. 2 - Vue sur l'église depuis la Montagne de Bard

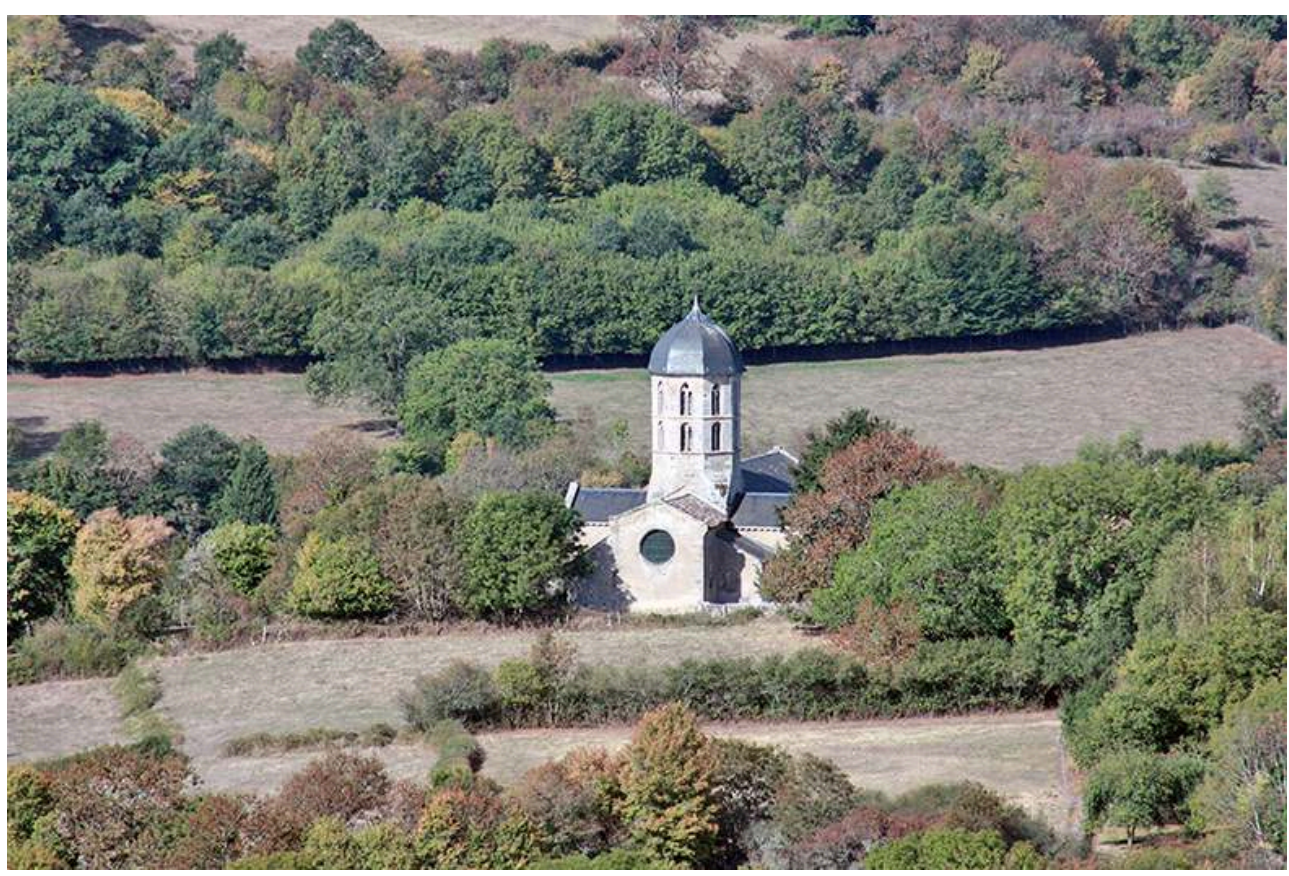

Cl. G. Pertuisot

3 Saint-Jean l'Évangéliste de Bard-le-Régulier est en fait l'unique témoin d'un ensemble prieural, aujourd'hui disparu ${ }^{1}$. Fondé au début du XII siècle par le comte de Nevers, ce prieuré abritait des chanoines «pauvres » qui suivaient la règle de saint Augustin ${ }^{2}$. Il dépendait de Saint-Germain-en-Brionnais, où l'évêque d'Autun Aganon avait institué, vers 1070-1085, cet ordre canonial réformé.

4 L'église présente un plan en croix avec une nef de quatre travées, voûtée en berceau brisé, un transept saillant et un chœur de deux travées voûtées en berceau plein cintre, flanquées de deux chapelles rectangulaires. Si l'édifice date du XII ${ }^{\mathrm{e}}$ siècle, le clocher a, quant à lui, été élevé au début du XIII ${ }^{\mathrm{e}}$ siècle. Parmi les vestiges témoignant de son importance passée, on note l'autel sur quatre colonnettes de la fin $\mathrm{du}_{\mathrm{XII}}{ }^{\mathrm{e}}$ ou du $\mathrm{XIII}^{\mathrm{e}}$ siècle, un ensemble remarquable de trente stalles en bois du milieu du XIV ${ }^{\mathrm{e}}$ siècle ${ }^{3}$, décorées de scènes bibliques ou plus quotidiennes, ainsi qu'un gisant en pierre, également du XIV siècle (fig. 3 et 4 ). 
Fig. 3 - L'intérieur de l'église

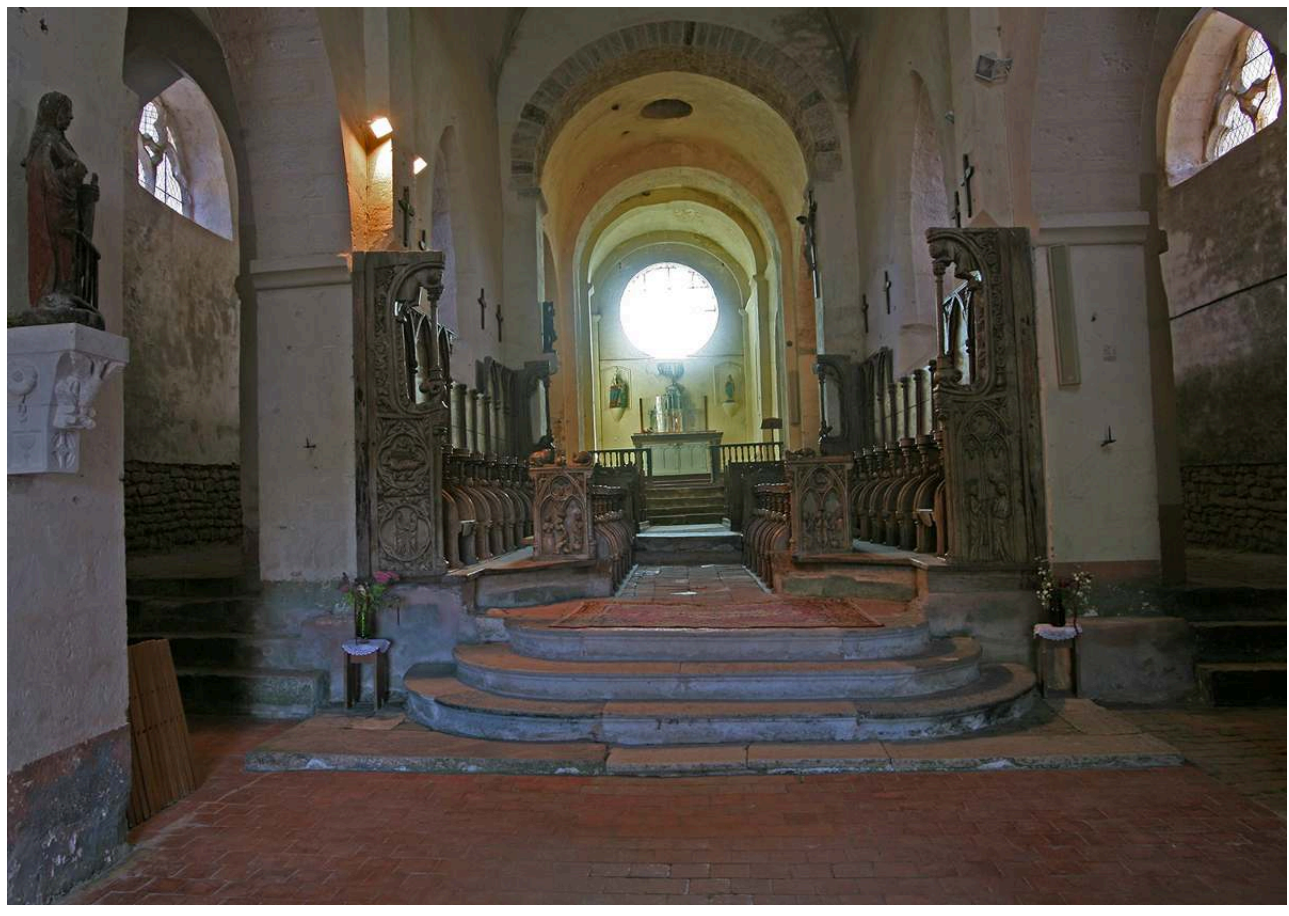

Cl. G. Pertuisot

Fig. 4 - Les stalles du XIV ${ }^{e}$ siècle

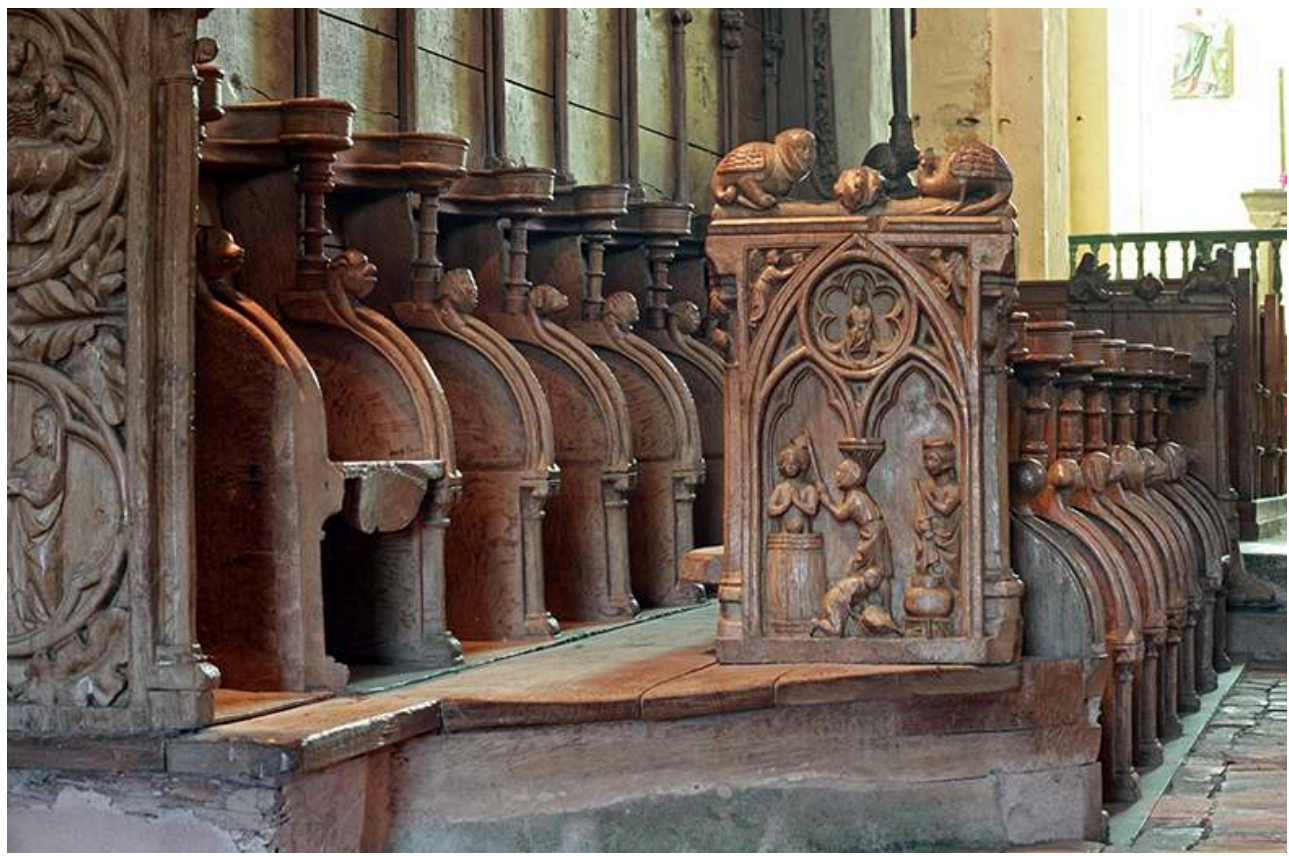

Cl. G. Pertuisot

5 Le prieuré ayant été réuni en 1724 au chapitre de Notre-Dame de Semur-en-Auxois, les religieux ont quitté les lieux en $1725^{4}$. Les bâtiments prieuraux, alors à l'abandon ${ }^{5}$, ont été démolis au XVIII ${ }^{e}$ siècle. 
6 L'église, devenue paroissiale, a été classée au titre des Monuments historiques le 10 décembre 1907. L'architecture vernaculaire du village, constituée de fermes de granit, calcaire et grès, comporte plusieurs remplois visibles provenant à l'évidence du prieuré démantelé. Une colonne, dont la provenance exacte n'est pas connue, mais qui appartenait sans doute à l'ensemble architectural disparu, est également exposée sur le parvis de l'église (fig. 5).

Fig. 5 - La colonne exposée sur le parvis de l'église

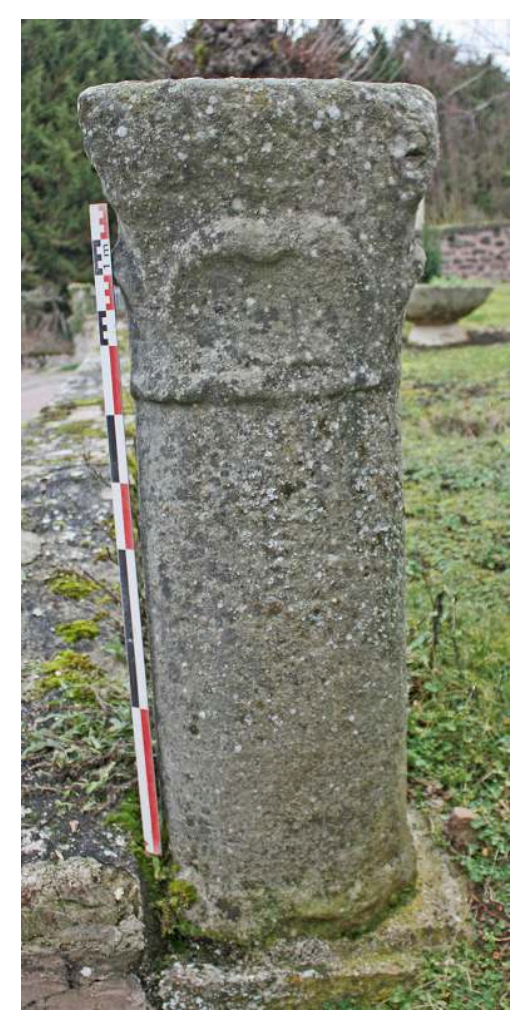

Cl. G. Pertuisot

\section{L'opération archéologique}

\section{Les fenêtres d'observation}

7 Des travaux de drainage et de restauration ont été projetés pour l'église en proie à l'humidité. Un diagnostic archéologique de quatre jours a donc été prescrit par le Service régional d'archéologie de Bourgogne-Franche Comté afin de récolter les données avant leur potentielle destruction.

8 L'implantation d'un réseau de drainage autour d'une église représente peu de surface, semble d'un impact minime. Pourtant elle est potentiellement particulièrement destructrice, puisqu'elle va couper l'édifice de ses liens stratigraphiques avec son environnement, bien souvent le cimetière. À Bard-le-Régulier, il s'agissait non seulement du cimetière, mais aussi des vestiges des bâtiments conventuels. Aussi quatre sondages ont été disposés autour de l'église, avec une attention particulière du côté sud, dont le mur porte encore les stigmates d'un bâtiment disparu avec la présence de corbeaux encastrés dans le mur gouttereau et le transept. 
9 Un premier sondage, sur le parvis de l'église (sondage 1), s'est avéré pauvre en information, si ce n'est la découverte de niveaux successifs de remblais sur une épaisseur de plus d'1 m, comportant de manière épisodique des ossements humains, dont la présence est liée sans doute au remaniement du cimetière situé à proximité.

10 Un autre sondage (sondage 4), au droit du mur du collatéral nord, a été infructueux. Cette zone, située entre l'église et le cimetière, a été curée jusqu'à la roche à l'occasion de la création d'une cour anglaise au début $\mathrm{du} \mathrm{xx}^{\mathrm{e}}$ siècle, dans le but sans doute déjà d'assainir l'édifice en proie aux infiltrations d'humidité (fig. 6). Les relations entre l'édifice et le cimetière originel ont donc été perdues.

Fig. 6 - La cour anglaise longeant l'édifice côté nord

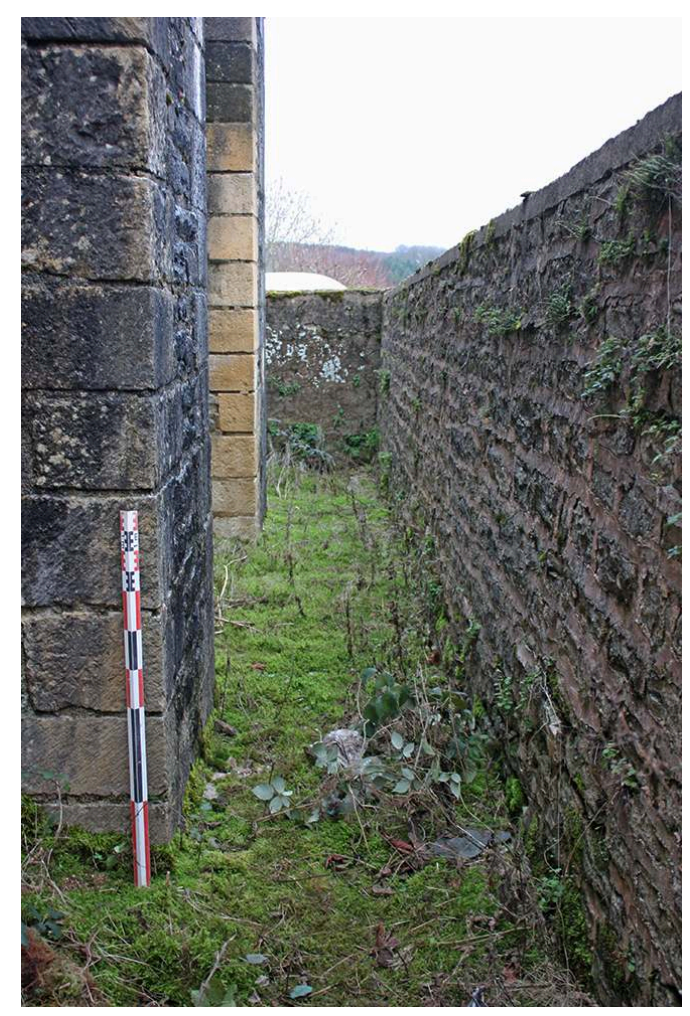

Cl. G. Pertuisot

11 Les deux autres sondages, situés sur le flanc sud de l'église, ont livré davantage d'informations (fig. 7 et 8). 
Fig. 7 - Localisation des sondages autour de l'église avec plan et coupes du sondage 2
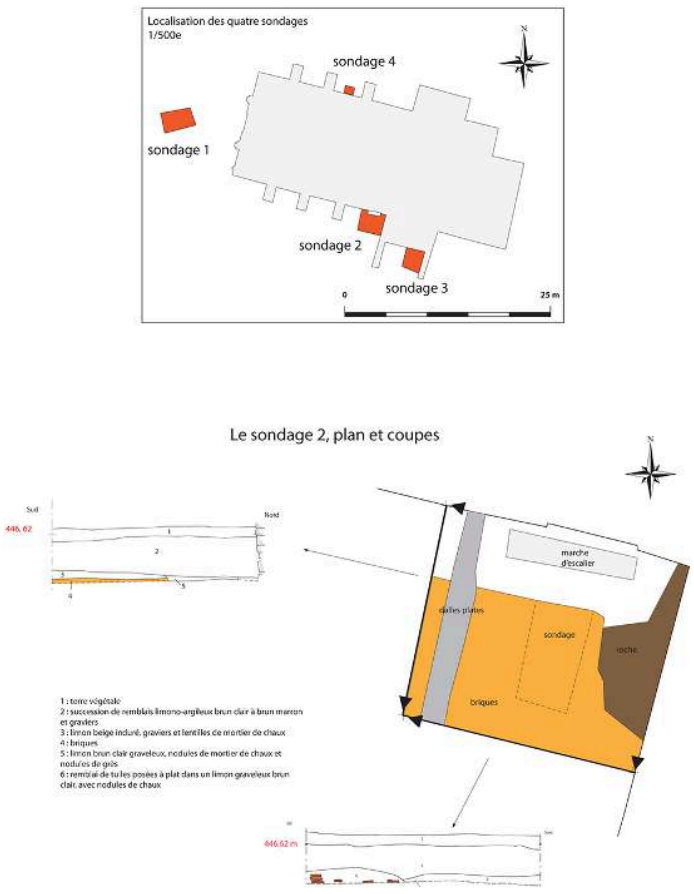

Levé topographique M. Lagache, DAO C. Méloche et G. Pertuisot.

Fig. 8 - Le sol de tomettes en terre cuite, décoré d'une ligne de dalles calcaires

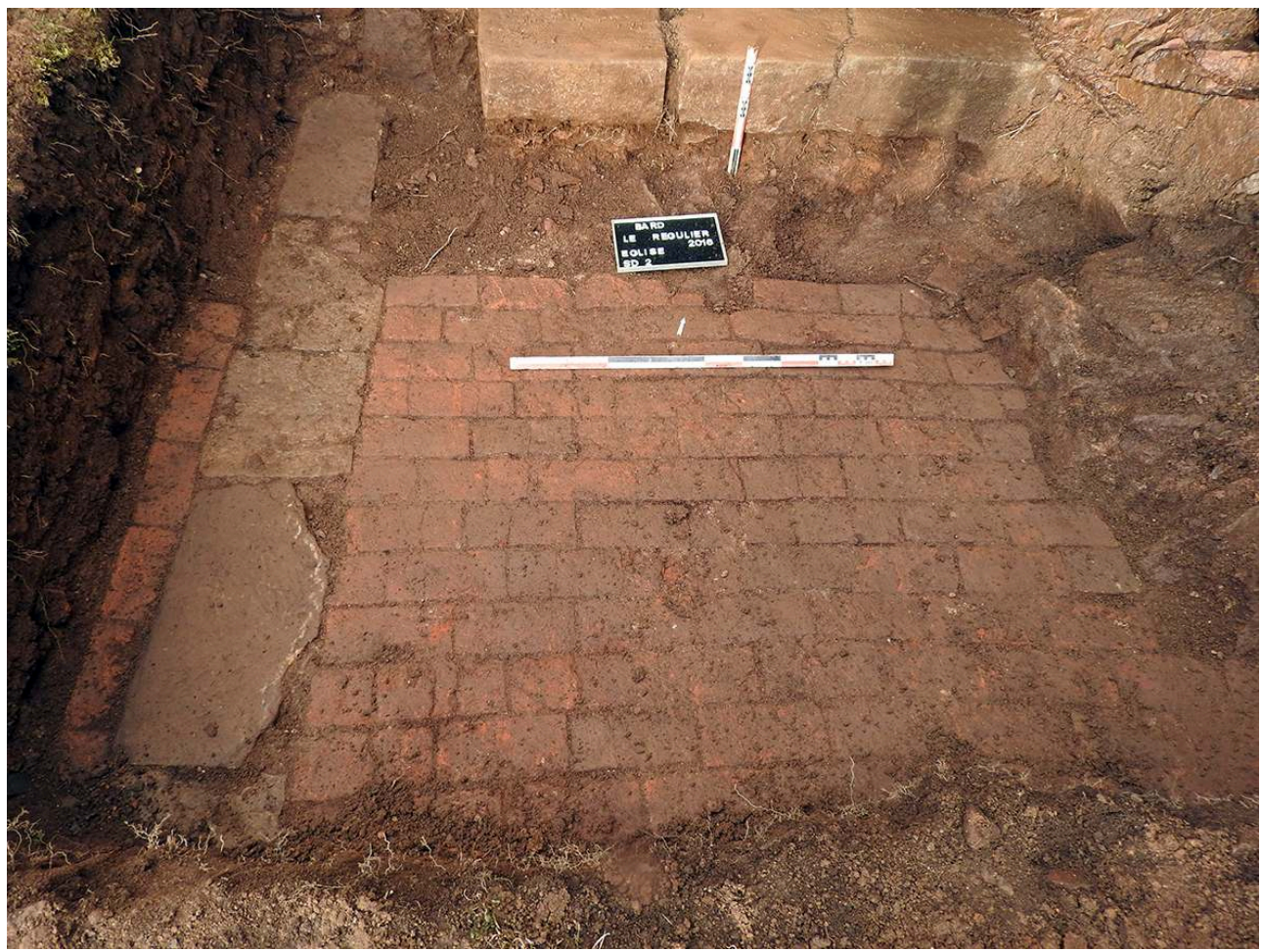

Cl. G. Pertuisot 

dalles calcaires, parfaitement conservé. Les briques de pavement ont des dimensions différentes suivant leur positionnement; les dalles calcaires forment une limite entre deux types de briques. On perçoit clairement un ordonnancement des matériaux dans un but esthétique et sans doute fonctionnel. La datation des vestiges s'est avérée problématique. En effet, seul un tesson de céramique glaçurée, présentant un faciès du XVIII siècle, a été observé dans l'unité stratigraphique de scellement du sol. Un petit test manuel a été effectué dans le niveau de briques afin d'appréhender d'éventuels niveaux antérieurs. Force a été de constater que celui-ci reposait directement sur le substrat géologique.

Situé contre le bras du transept, entre les deux contreforts, le sondage 3 a révélé des niveaux de remblais et de démolition sur $0,50 \mathrm{~m}$ d'épaisseur, avec des fragments de terre cuite, des ossements divers (humains ou non) et un élément sculpté en calcaire à décor végétal, témoin de la richesse passée de l'ensemble architectural (fig. 9).

Fig. 9 - Élément architectural sculpté issu des remblais du sondage 3 effectué entre les deux contreforts adossés au bras du transept

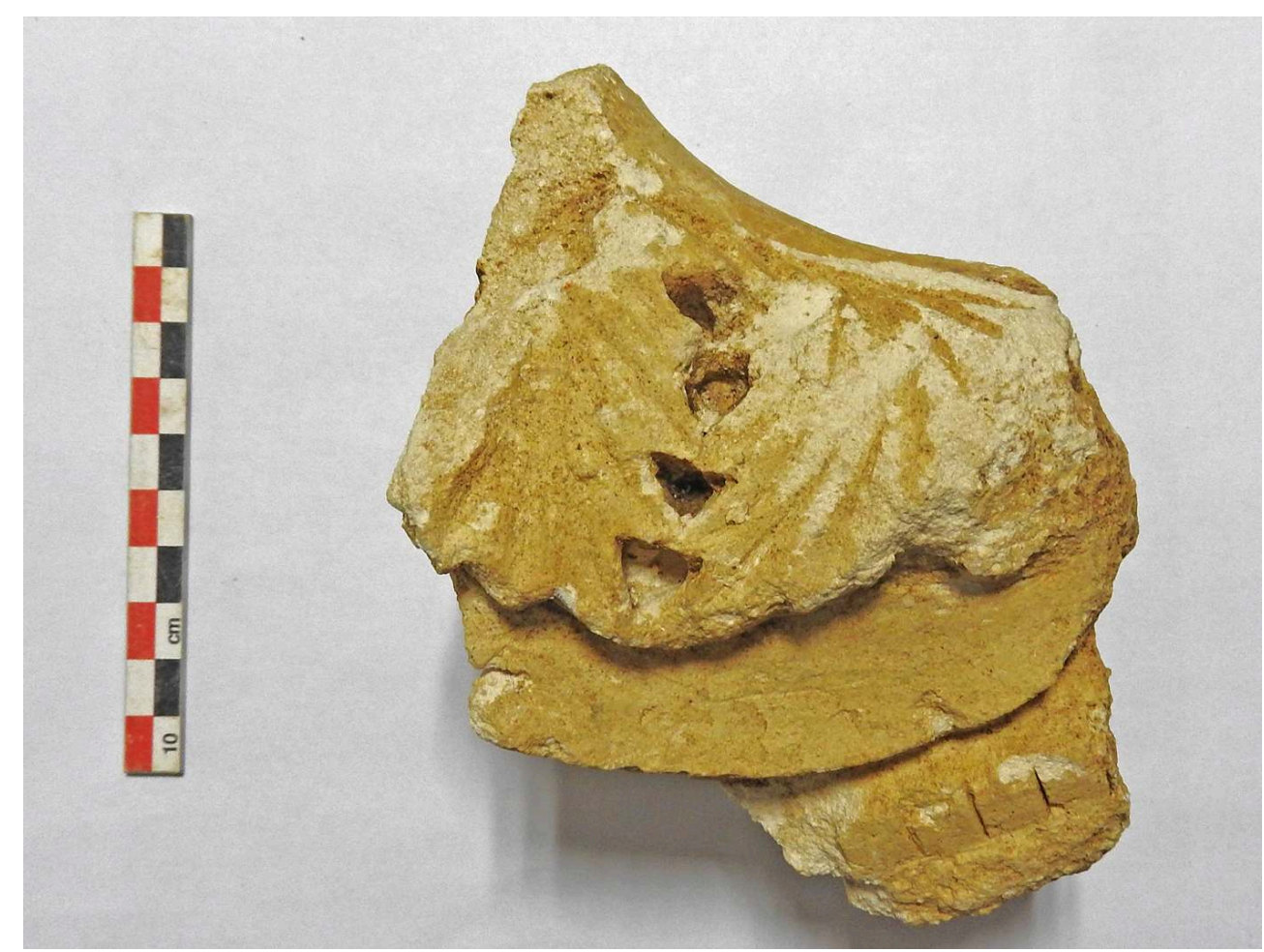

Cl. G. Pertuisot

Le sondage a été poursuivi jusqu'à atteindre une couche de démolition puis un niveau de piétinement noir compact, à $0,85 \mathrm{~m}$ de profondeur, testé sur $0,10 \mathrm{~m}$ d'épaisseur, permettant de récolter des tessons de céramique noire, et d'autres glaçurés des XIVe $\mathrm{XV}^{\mathrm{e}}$ siècles.

15 L'étroitesse des sondages autorise difficilement une interprétation poussée, mais les éléments observés permettent d'évoquer une occupation de ces niveaux dès le XIV ${ }^{e}$ siècle et ce jusqu'au XVIII ${ }^{e}$ siècle. 


\section{Les documents d'archives}

Deux beaux documents, conservés aux Archives départementales de la Côte-d'Or, témoignent de l'importance du prieuré à l'époque moderne: tout d'abord une perspective cavalière de 1669 (fig. 10 et 11) et, ensuite, un plan du XVIII e siècle (fig. 12), avec mention des fonctions des bâtiments du prieuré.

Fig. 10 - Le plan de 1669 (ADCO, G 3344 : «Plan de l'enclos du prieuré avec le champ de la vigne »)

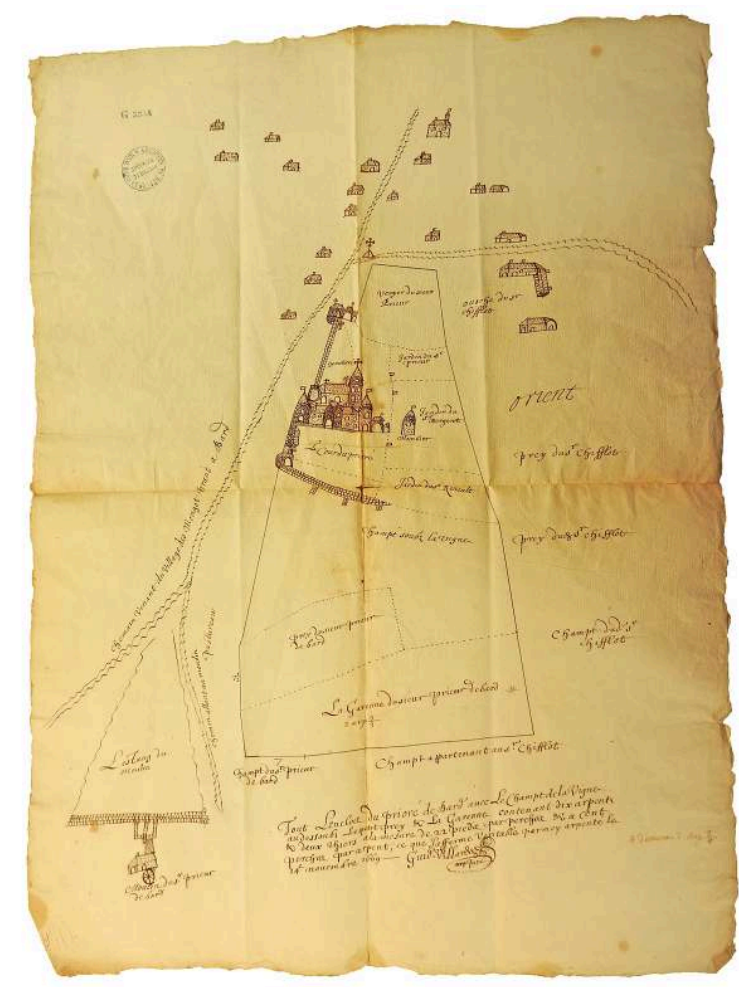

$0,44 \times 0,60 \mathrm{~m}$

Cl. G. Pertuisot 
Fig. 11 - Détail du plan de 1669

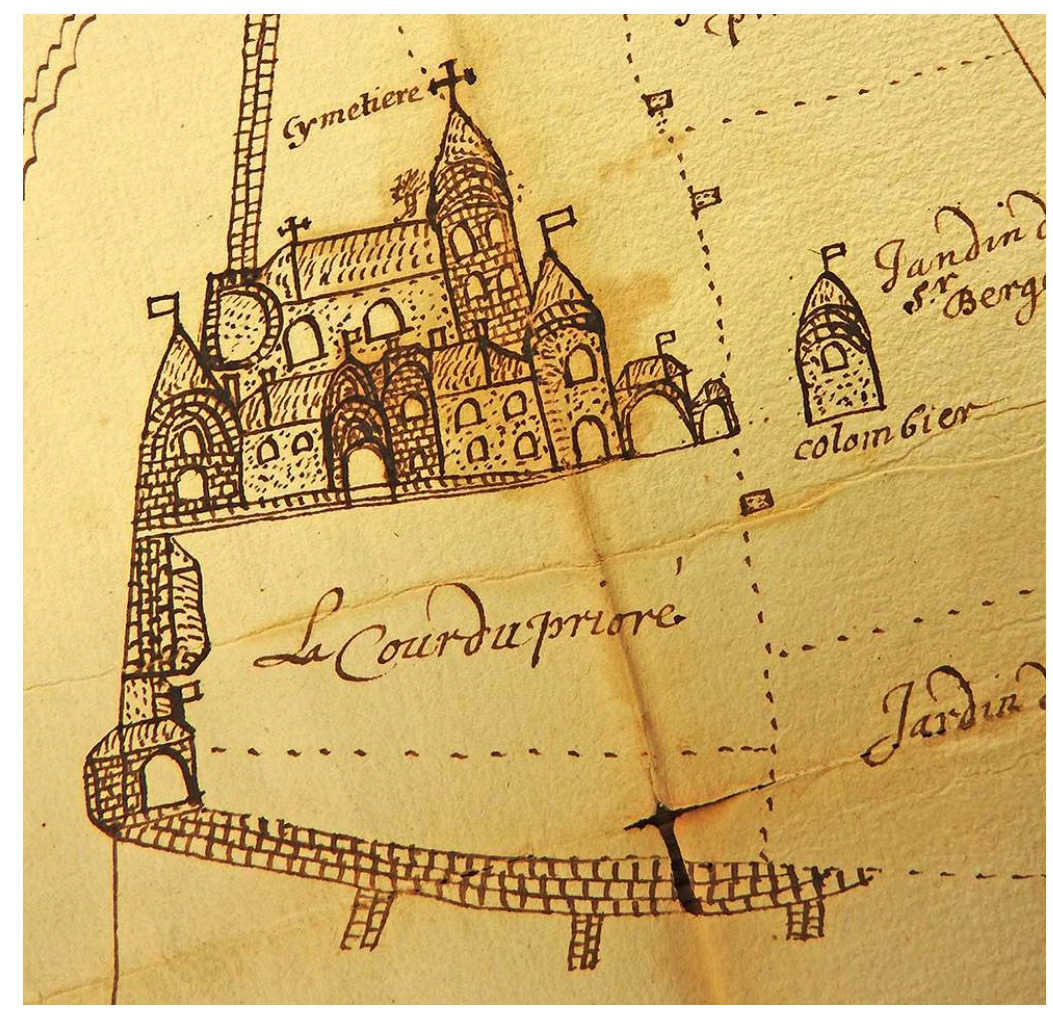

Cl. G. Pertuisot 
Fig. 12 - Le plan terrier du XVIII ${ }^{\mathrm{e}}$ siècle (ADCO, G 3345 : «Plans détaillés du territoire de Bar, village, parcelles numérotées avec nom des propriétaires ")

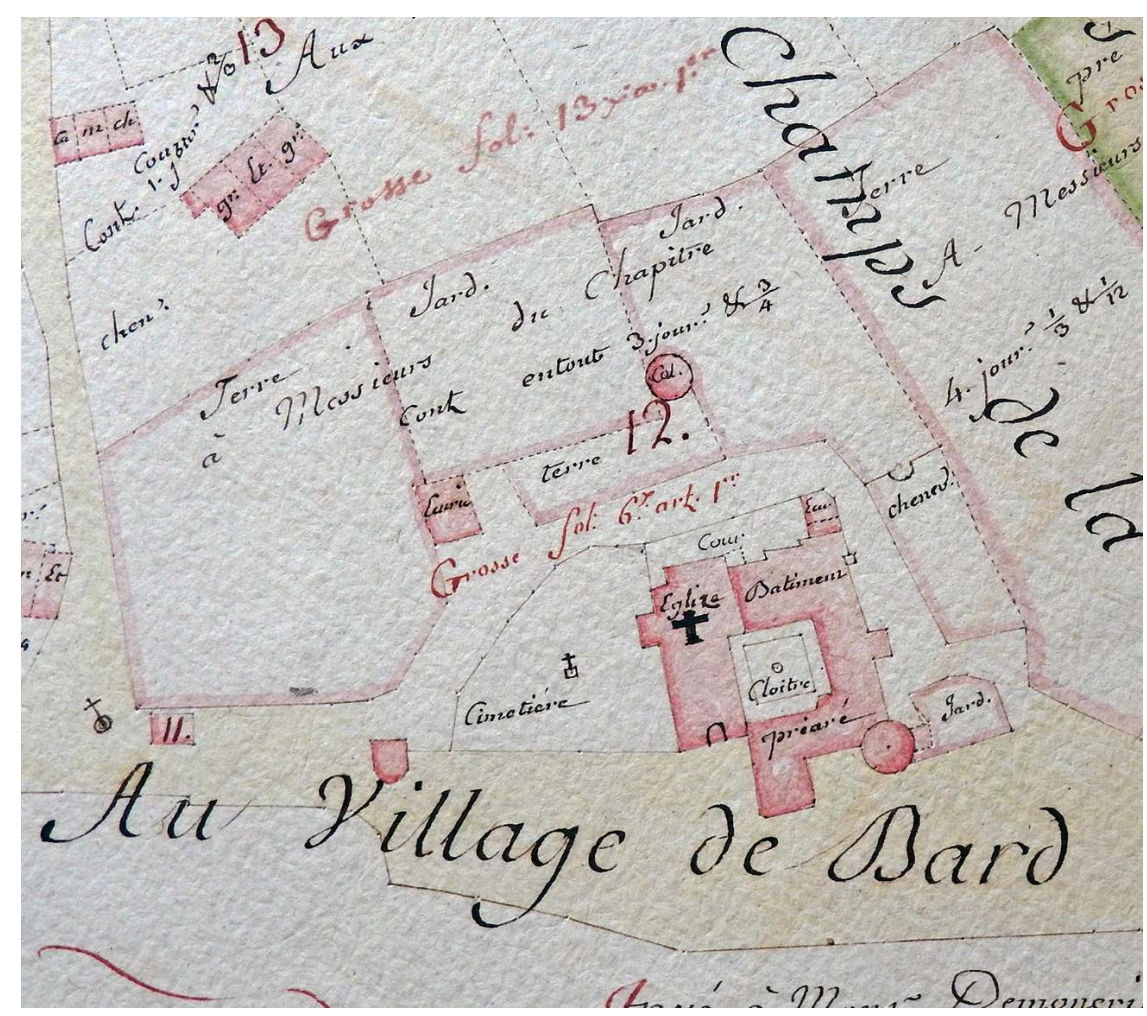

$0,90 \times 0,60 \mathrm{~m}$

Cl. G. Pertuisot

17 Si la perspective cavalière de 1669 évoque plus un ressenti, avec la figuration des matériaux, la hauteur des élévations, la quantité d'ouvertures dans les bâtiments, la disproportion de l'aspect massif de l'ensemble prieural face à l'habitat dispersé du village, le plan du XVIII ${ }^{\mathrm{e}}$ siècle renvoie à l'organisation des bâtiments et met en exergue le cloître, qui n'était pas perceptible sur le document de 1669. L'église y est figurée, mais aussi les bâtiments prieuraux, une tourelle, un colombier, un mur d'enclos ainsi que de nombreux jardins et terres cultivées.

Avec les réserves qui s'imposent, ces archives constituent des documents précieux et leur superposition avec le plan des découvertes archéologiques est particulièrement instructive (fig. 13). 
Fig. 13 - Superposition du plan effectué au cours du diagnostic avec le plan terrier du XVIII siècle

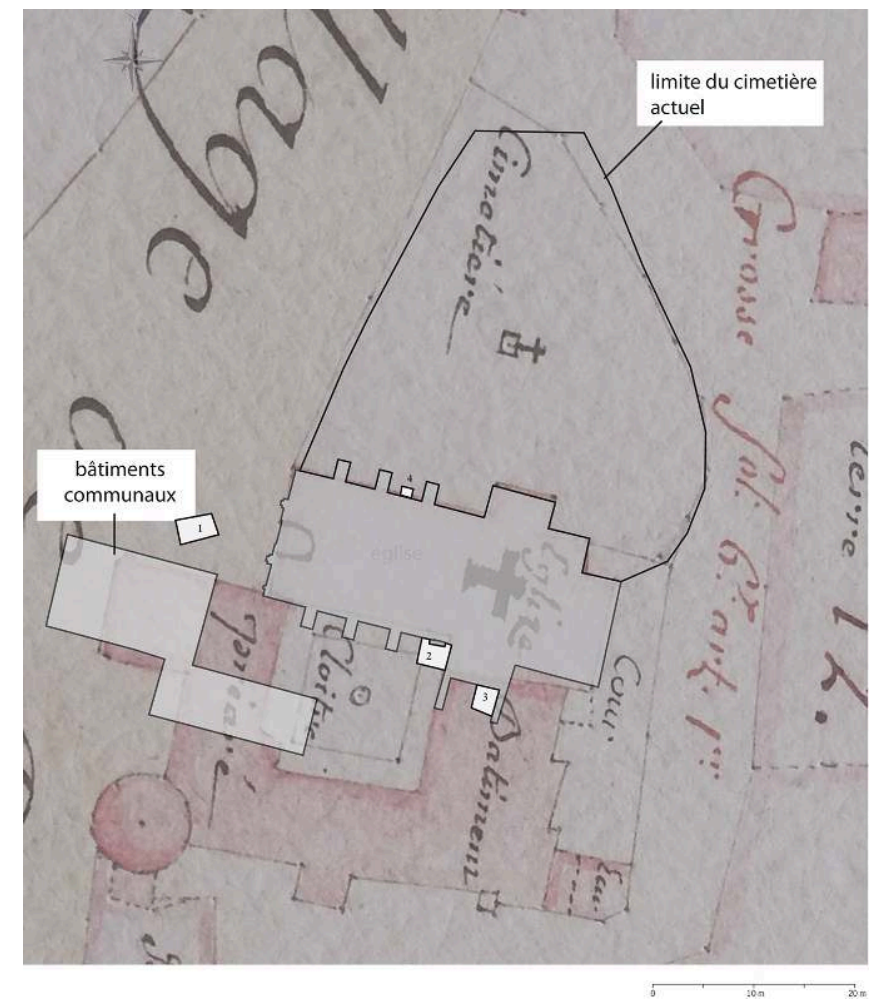

DAO G. Pertuisot

19 Le sol de briques du sondage 2 peut ainsi être aisément interprété : il s'agit du niveau de circulation de la galerie du cloître, tel qu'il apparaît sur le plan du XvIII ${ }^{\mathrm{e}}$ siècle.

Le corbeau qui surplombe le sondage est situé à 3,08 $\mathrm{m}$ au-dessus du sol d'origine du cloître. On imagine alors qu'il a pu supporter la charpente d'un bâtiment doté d'un étage, comme le suggère la vue cavalière de 1669 , avec ses deux rangées de fenêtres. L'escalier situé à l'aplomb du sondage pouvait alors mener de l'église au cloître, même si les traces de boucharde sur le chambranle de la porte laissent penser que celle-ci n'est pas antérieure au XVIII ${ }^{e}$ siècle ou qu'elle aurait été remaniée a posteriori.

Les deux contreforts adossés au bras du transept - le contrefort ouest laissant par ailleurs apparaître une baie rebouchée - s'avèrent être les vestiges des murs d'un bâtiment anciennement accolé au cloître (fig. 14). 
Fig. 14 - Départ de baie dans le contrefort

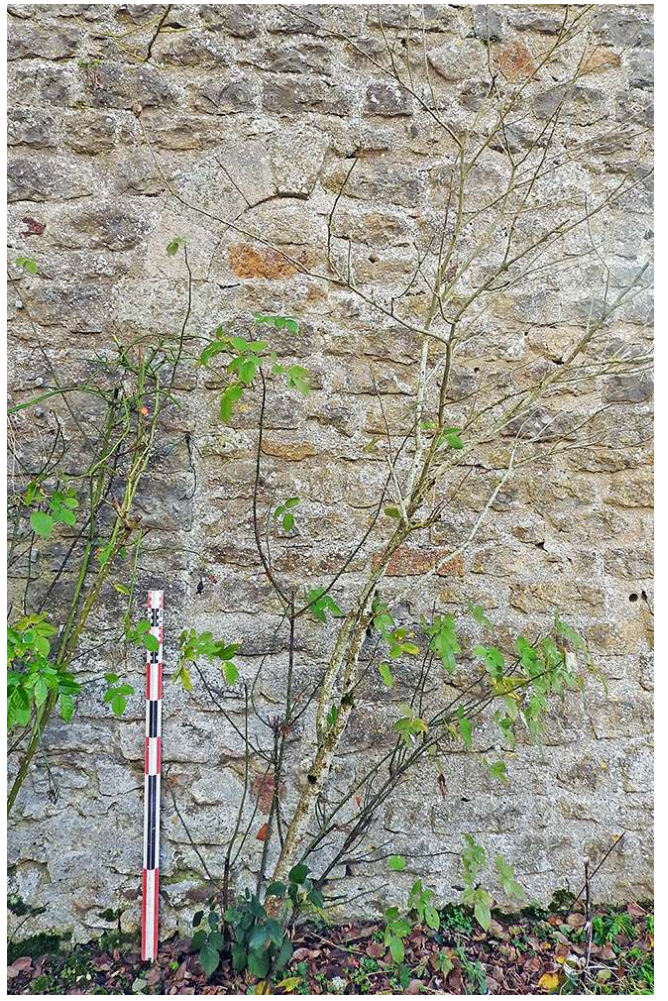

Cl. G. Pertuisot

22 Ces éléments maçonnés n'ont sans doute pas été démantelés entièrement afin de préserver la stabilité de l'église, qui devait demeurer après démolition du prieuré.

23 À ces deux plans s'ajoute celui du cadastre napoléonien dressé en 1842 (fig. 15). 
Fig. 15 - Détail du cadastre napoléonien de 1842

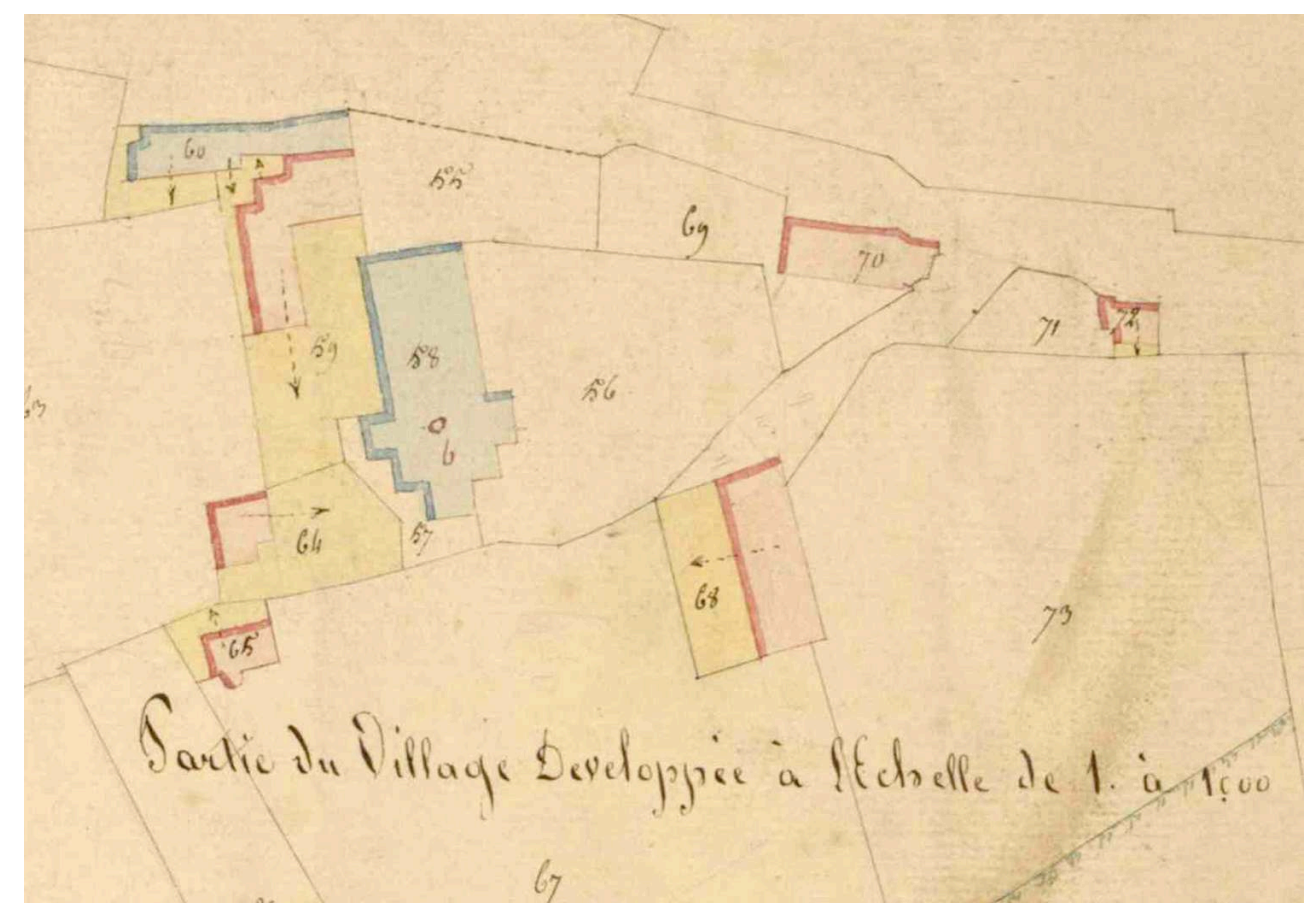

www.archives.cotedor.fr

Le prieuré n'y figure plus, il a disparu du paysage de Bard-le-Régulier, ne se laissant deviner que par quelques vestiges de murets d'enclos délimitant les parcelles.

\section{Conclusion}

À Bard-le-Régulier, la confrontation des données de fouille et des plans anciens a permis d'appréhender différemment un simple diagnostic archéologique. Des renseignements fonctionnels sur la topographie et l'organisation même de l'ensemble prieural viennent, en effet, compléter et abonder les informations métriques révélées par le terrain - profondeur des vestiges, observations du bâti -, mais aussi les données liées aux artefacts mis au jour - typo-chronologie céramique par exemple, matériaux de construction -, en apportant un éclairage inédit.

Ce type d'opération, bien que modeste par sa durée (4 jours) et par la surface couverte par les sondages - l'emprise de la tranchée de drainage -, représente une occasion rare pour les archéologues d'accéder à des terroirs ruraux très peu concernés par des travaux déclenchant des surveillances archéologiques préventives.

Ce fut également, dans le cas de l'opération menée à Bard-le-Régulier, la possibilité pour nous d'observer les vestiges d'un patrimoine religieux omniprésent du Moyen Âge jusqu'à l'époque moderne, pour lesquels avaient été dressés des documents graphiques, qui se sont avérés très parlants et dont la publication permet ici d'assurer la pérennité. Reçu : 28 février 2018 - Accepté : 18 octobre 2018 


\section{NOTES}

1. S. BERTRAND, L'ancien prieuré de Bar-le-Régulier, Liernais, 2008.

2. Il s'agissait de chanoines réguliers demeurant dans le siècle, mais ayant renoncé à la propriété personnelle au contraire d'autres collèges de chanoines.

3. А. colombet, L'église de Bar-le-Régulier (Côte-d'Or) et ses stalles du XIVee siècle, Commission d'art sacré du diocèse, [Dijon], 1990 ( $3^{\mathrm{e}}$ éd.).

4. S. BERTRAND, L'ancien prieuré..., op. cit.

5. En témoignent les comptes rendus de visite conservés aux Archives départementales de la Côte-d'Or (G 3326 et G 3330).

\section{INDEX}

Mots-clés : Moyen Âge central, époque moderne, église paroissiale, prieuré, archives

\section{AUTEUR}

\section{GAËLLE PERTUISOT}

Responsable de recherche archéologique pour le Moyen Âge, Inrap Bourgogne-Franche-Comté (centre de Dijon) 